Spreading resistance at the nano-scale studied by scanning tunneling and field emission spectroscopy

Prabhava S. N. Barimar, Borislav Naydenov, Jing Li, and John J. Boland

Citation: Appl. Phys. Lett. 110, 263111 (2017); doi: 10.1063/1.4990392

View online: http://dx.doi.org/10.1063/1.4990392

View Table of Contents: http://aip.scitation.org/toc/apl/110/26

Published by the American Institute of Physics AIP $\mid \begin{aligned} & \text { Applied Physics } \\ & \text { Letters }\end{aligned}$

Save your money for your research.

It's now FREE to publish with us -

no page, color or publication charges apply. 


\title{
Spreading resistance at the nano-scale studied by scanning tunneling and field emission spectroscopy
}

\author{
Prabhava S. N. Barimar, Borislav Naydenov, Jing Li, and John J. Boland ${ }^{\text {a) }}$ \\ School of Chemistry, Centre for Research on Adaptive Nanostructures and Nanodevices, \\ Trinity College Dublin, Dublin, Ireland
}

(Received 10 April 2017; accepted 14 June 2017; published online 28 June 2017)

\begin{abstract}
We explore the capability of scanning tunneling microscopy (STM) and scanning tunneling spectroscopy (STS) to study nanoscale $\mathrm{Si}(100)$ device layers in silicon-on-insulators (SOIs). These device layers are a macroscopic 2D silicon sheet, and understanding the effective coupling of charge in and out of this sheet allows the determination of whether it is possible to accurately measure the electronic properties of the sheet. Specifically, we examine how the spreading resistance is manifested following the processing of SOI device layers with various doping levels. Depending on the doping level, ultra-thin SOI can exhibit significant blue shifts of the peaks in the tunneling and field emission spectra. By comparing these peak shifts with the film resistivity, it is possible to estimate the contribution of the spreading resistance in STM and STS. We show that STM can be used to study the effective n-type dopant concentrations in the $10^{13}-10^{16} \mathrm{~cm}^{-3}$ range. Furthermore, we demonstrate that with a sufficiently high doping level, $5 \mathrm{~nm}$ thick SOI devicelayers can be measured and exhibit bulk like electronic characteristics. Published by AIP Publishing. [http://dx.doi.org/10.1063/1.4990392]
\end{abstract}

Silicon-on-Insulators (SOIs) have attracted a great deal of scientific interest as they offer the opportunity to study nanoscale devices and electron confinement effects. ${ }^{1}$ The device layer [Fig. 1(b)] in SOIs is essentially a macroscopic near-perfect 2D silicon sheet. The use of SOIs in microelectronics is becoming prevalent, and many of the expected advances in the device technology depend essentially on attaining increasingly thinner device layers. ${ }^{1}$ Due to charge traps at the device layer and buried oxide (BOX) interface, thinner SOIs tend to be depleted of charge carriers, thus affecting the transport properties. ${ }^{1-3}$ Depleted and heavily thinned SOIs are difficult to be characterized by scanning tunneling microscopy (STM) due to charging. ${ }^{2,3}$ By heavily doping $\left(\sim 10^{19} \mathrm{~cm}^{-3}\right)$ the device layer, the carrier depletion in the layer can be reduced, and STM measurements may be carried out even at liquid nitrogen temperature, but these high doping levels are not of interest for device applications.

Before STM and scanning tunneling spectroscopy (STS) can be used to characterise nanoscale devices, it is critical to understand the influence of doping on the fundamental operation of STM. In normal STM operation, the tunnel gap resistance dominates, and the sample resistance and spreading resistance play a negligible role. ${ }^{4}$ Consequently, the applied bias allows the determination of energy states around Fermi energy $\left(\mathrm{E}_{\mathrm{F}}\right)$ - the very basis of STS. The spreading resistance can arise due to the small area of the tunneling contact and also depends on the sample resistivity. ${ }^{5}$ When the resistivity of the sample is high, the spreading resistance can become comparable with the tunneling resistance, ${ }^{4}$ leading to a voltage drop within the sample that can be observed in both STM and STS. Also, in the case of semiconductors, the high field from the STM tip can penetrate into the sample and lead to a tip field induced band bending in the semiconductor.

a)Author to whom correspondance should be addressed: jboland@tcd.ie.
Consequently, it is expected that an interplay between the spreading resistance and band bending has the potential to affect the measured properties of SOI device layers. ${ }^{4,6-8}$ Tipfield induced band bending in a $\mathrm{Si}(100)$ device layer is limited to a maximum of $0.8 \mathrm{eV}$ due to the Fermi level pinning of the surface state. ${ }^{9}$ However, contribution from the spreading resistance can in principle give rise to much higher voltage drop. ${ }^{9}$ The spreading resistance adds up in series ${ }^{4}$ with the tunneling resistance and leads to a drop in the applied voltage in the sample near the tip location. This local voltage drop in the device layer will shift the peaks in the tunneling and field emission spectra.

This article discusses the sensitivity of STM, STS, and field emission spectroscopy to the spreading resistance in SOI device layers and thus to the effective resistivity of the subsurface substrate volume with nanometre-scale lateral spatial resolution. This article shows that STM and STS can also be used to study the resistivity profile in semiconductors with high spatial resolution. Scanning spreading resistance microscopy (SSRM) is a powerful contact mode spreading resistance technique used for mapping the local dopant profile and electrical properties with nanometer-scale spatial resolution. ${ }^{10}$ Lately, SSRM has become a standard technique to study the nanoscale resistivity profile in semiconductors, ${ }^{11}$ especially the semiconductor nanostructures. ${ }^{12}$ However, the non-contact nature of STM reduces the non-linear effects ${ }^{13}$ arising due to the tip-surface interaction. Additionally, we demonstrate that by highly doping the ultra-thin SOI, the effects of tip induced band bending and spreading resistance can be significantly reduced to obtain bulk-like device layers with thicknesses down to $5 \mathrm{~nm}$.

All STM measurements were performed using a Createc low temperature STM (LT-STM) operating at liquid nitrogen temperature in an ultra-high vacuum (UHV) environment with a base pressure lower than $1 \times 10^{-11}$ mbar. A $45 \mathrm{~nm}$ 


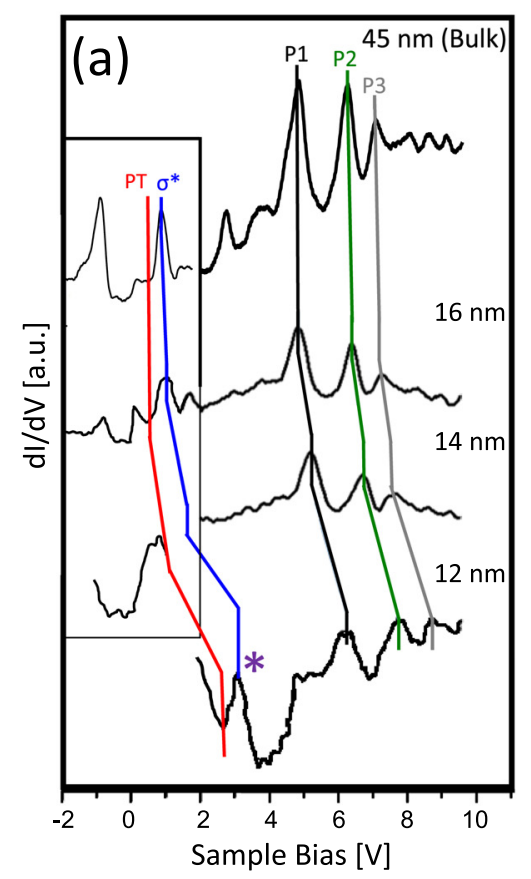

(b)

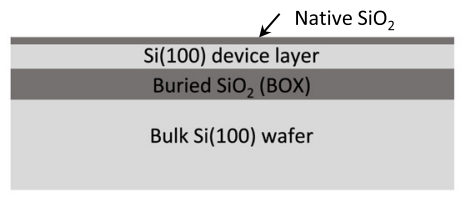

(c)

(d)
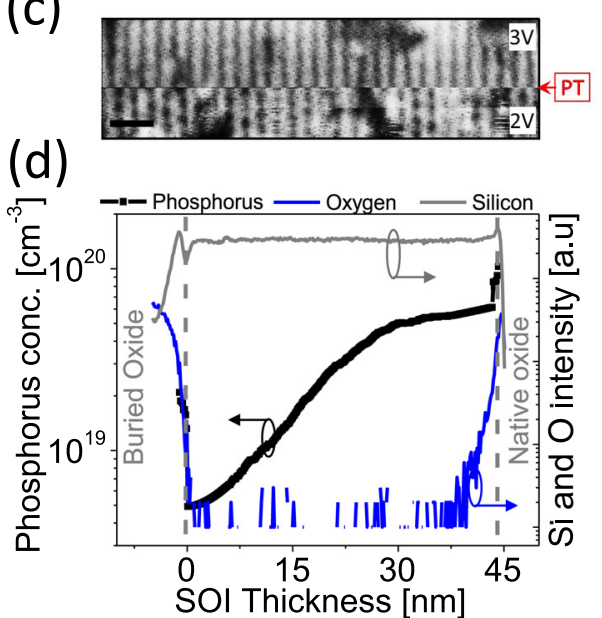

FIG. 1. (a) Tunneling spectra (IV) and field emission $[z(V)]$ spectra of the thinned SOI showing peak shifts. (b) Schematic of the SOI structure. (c) 'Topographic phase transition' (PT) between 3 and $2 \mathrm{~V}$ (setpoint current $=20 \mathrm{pA}$ ) for the $12 \mathrm{~nm}$ SOI. The scale bar in the image is $2 \mathrm{~nm}$. (d) Phosphorus conc. (black curve) recorded with SIMS depth analysis. Oxygen (blue) and silicon (grey) profiles are also shown for reference.
SOI doped with phosphorous to $\sim 10^{19} \mathrm{~cm}^{-3}$ was used to study the spreading resistance effects. Further, measurements were also carried out on a $<12 \mathrm{~nm}$ SOI with doping up to $\sim 10^{20} \mathrm{~cm}^{-3}$. Before loading into the UHV chamber, samples were subjected to an ex-situ cleaning process which involved oxygen plasma treatment and hydrogen fluoride (HF) rinse. ${ }^{14}$ A prolonged oxygen plasma treatment followed by HF rinse was used to thin the SOI (the quality of the thinned SOI is shown in Fig. S1 in the supplementary material), and the thickness was measured with an accuracy of $\pm 0.5 \mathrm{~nm}$ with a J. A. Woollam Alpha-SE ellipsometer calibrated by cross sectional transmission electron microscopy (TEM) analysis. The dopant concentration and resistivity profile in the device layer were characterized with secondary ion mass spectroscopy (SIMS) and 4-point probe electrical resistivity measurements. The contact potential difference (CPD) and work function of $\mathrm{H}$-passivated SOIs were measured with respect to a reference $\mathrm{Au}$ layer using a Bruker Dimension atomic force microscope at room temperature in the kelvin probe microscopy (KPM) mode.

Samples were flash annealed at around $1020 \mathrm{~K}$ to desorb oxide and form a sharp $2 \times 1$ Low-Energy Electron Diffraction (LEED) pattern at room temperature. Electrochemically etched tungsten tips inked with Pt were used for STM and STS measurements. ${ }^{15}$ The quality of the tips was verified on a sputtercleaned $\operatorname{Pt}(111)$ crystal before and after characterization of SOIs to ensure that the probe was not modified. Tunneling and field emission spectra were obtained using STS techniques including field emission spectroscopy ${ }^{16}$ and variable height spectroscopy. ${ }^{15}$

Figure 1(a) shows both the tunneling and field emission spectra obtained from the $45 \mathrm{~nm}$ device layer, along with samples made from the same SOI thinned down to 16,14 , and $12 \mathrm{~nm}$. For the $12 \mathrm{~nm}$ sample, it is impossible to record the tunneling spectra due to the instability of the current at low biases. In all cases, emission spectra recorded at biases above $4 \mathrm{~V}$ reveal a systematic shift in the peak positions (indicated with lines marked as P1, P2, and P3), as the device layer thickness is decreased. In addition, it is possible to exploit the change in STM imaging contrast at $+0.5 \mathrm{~V}$ and $+1 \mathrm{~V}$ on a $\mathrm{Si}(100)(2 \times 1)$ surface that results from predominant tunneling into the $\pi^{*}$ dangling bond states (located on the dimers) or back bond $\sigma^{*}$ states (located between dimers), respectively. ${ }^{17}$ Switching the STM voltage set-point between the two voltages results in a change in image contrast [PT in Fig. 1(a)], which can also be used to track peak shifts as the film thickness is reduced. This image phasetransition (PT) for the $12 \mathrm{~nm}$ SOI is shown in Fig. 1(c) where it is observed between $2 \mathrm{~V}$ and $3 \mathrm{~V}$ (PT for the $14 \mathrm{~nm}$ SOI is shown in Fig. S2 in the supplementary material), consistent with the blue shift of the emission peaks for thinner samples. This observation clearly identifies the peak marked $\left(^{*}\right)$ for the $12 \mathrm{~nm}$ SOI as the $\sigma^{*}$ state peak. The shift in the image phase-transition voltage is indicated by the red line in Fig. 1(a) and marked as PT.

The dopant level of each film in Fig. 1(a) is reduced as the device layer is progressively thinned, and thus, the peak shifts in the STS data reflect the dopant profile within the original film. This is consistent with SIMS depth profile (DP) analysis for $\mathrm{Si}, \mathrm{P}$, and $\mathrm{O}$ for the original $45 \mathrm{~nm}$ thick device layer [Fig. 1(d)]. The grey dotted lines in the graph indicate the physical boundaries of the device layer. The dotted line on the left indicates the SOI/buried oxide (BOX) interface and the line on the right the surface with native oxide. The phosphorous (ion implanted, n-type dopant) concentration in the device layer of the SOI decreases significantly towards the $\mathrm{Si} / \mathrm{BOX}$ interface.

Figure 2(a) shows the measured voltage shifts observed in STM PT and STS measurements in Fig. 1(a) for different device layer thicknesses. The shifts observed for the peaks in field emission spectra, the $\sigma^{*}$ peak, and phase transition voltage all show a gradual increase down to $14 \mathrm{~nm}$, following which there is a sharp increase at $12 \mathrm{~nm}$ film thickness. A maximum voltage shift of about $1.5-2 \mathrm{~V}$ was observed for 
(a)

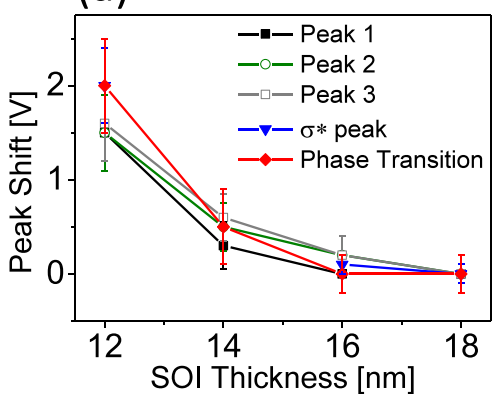

(b)

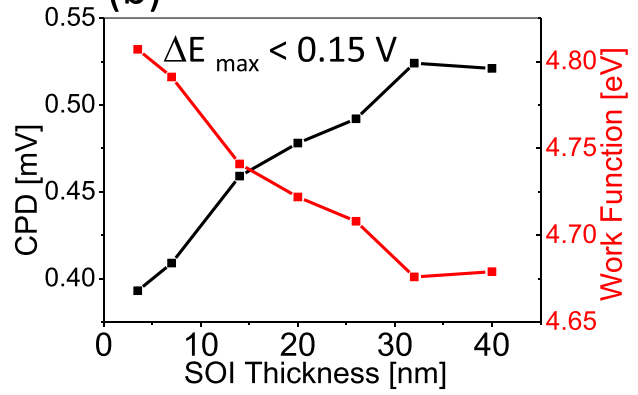

FIG. 2. (a) Voltage shift observed in STM and STS measurements as a function of SOI film thickness. Peaks 1, 2, and 3 are shown in Fig. 1(b) as P1, P2, and P3. (b) CPD (black) and work function (red) measured with ex-situ KPM on hydrogen passivated thinned SOIs plotted with respect to the thickness of the device layer. the $12 \mathrm{~nm}$ SOI. All tunneling and emission peaks in Fig. 2(a) experience similar blue shifts consistent with a translation of the entire spectrum. The first peak (P1) in the field emission spectra gives the approximate position of the work function of the surface. ${ }^{16,18}$ The work function of the film was measured independently via KPM of the hydrogen passivated device layer. Figure 2(b) shows the variation in the CPD and work function of the film as a function of thickness. There is a clear increase in the work function of the thinned SOI, indicating a lower n-type phosphorus dopant concentration, consistent with the phosphorous SIMS profile shown in Fig. 1(c). However, the variation in the work function, observed for the whole range of device-layer thicknesses, was less than $0.15 \mathrm{eV}$, in contrast to the $1.5-2 \mathrm{~V}$ shift observed in the case of STM and STS measurements. This indicates that the emission peaks shift shown in Fig. 2(a) cannot be solely explained by the work function changes caused by the dopant profile in the film.

STM can be significantly affected by the high electric field of the STM tip which can penetrate into a semiconductor sample. ${ }^{6,7,9,19}$ The penetrating field can lead to 3 dimensional tip induced band bending. ${ }^{4}$ The extent of bending is however determined by the carrier concentration in the semiconductor ${ }^{4}$ and can lead to significant shifts in the peaks observed in the STS spectra, ${ }^{9}$ especially in high resistivity samples. ${ }^{4,6,8,20}$ For a $0.1 \Omega \mathrm{cm}$ n-type $\mathrm{Si}(100)$, the presence of a tip with a radius of $10 \AA$, at an applied bias of $1.5 \mathrm{~V}$, will induce a band bending of $0.4 \mathrm{~V}$. ${ }^{9}$ However, the maximum band bending for a ntype $\mathrm{Si}(100)-(2 \times 1)$ surface can be $0.8 \mathrm{~V} .{ }^{9}$ The filled state $\pi$ and the empty state $\pi^{*}$ bands on the $(2 \times 1)$ surface are located at $0.2 \mathrm{eV}$ below the valance band maximum (VBM) and $0.4 \mathrm{eV}$ below the conduction band minima (CBM), respectively, ${ }^{21}$ which constraints the $\mathrm{E}_{\mathrm{F}}$ value at the surface between the VBM and the empty $\pi^{*}$ state. The electric field from the tip with a positive bias can locally unpin the surface and bend the band until the Fermi level meets the VBM, resulting in a maximum bending of $+0.8 \mathrm{~V}$. In contrast, a negative bias on the tip results in just a small shift of $+0.09 \mathrm{~V}$ as the $\mathrm{E}_{\mathrm{F}}$ value is pinned at the bottom of the $\pi^{*}$ state. $^{9}$ However, the shift observed in Fig. 2(a) is much larger than the possible effects due to tip induced band bending. Additionally, a tip-induced field effect would show a strong bias dependence of the peak-shift in the field emission spectra since for example, the tip-induced electric field at $6 \mathrm{~V}$ is twice that at $2 \mathrm{~V}$. This is not observed in Fig. 1(a), which indicates a lack of band bending effects in this bias range.

Figure 3(a) shows the phosphorus dopant concentration profile in the device layer measured by SIMS and estimated from electrical resistivity at 300 and $77 \mathrm{~K}$. The electron mobility values were obtained from Semiconductor devices, by Sze. ${ }^{22}$ While SIMS shows the chemical concentration of phosphorus in the device layer, resistivity reflects the active dopant concentration in the film. ${ }^{23}$ Resistivity measurements, however, are influenced by the depletion caused by the buried oxide interface traps, which is typically around $10^{11} \mathrm{~cm}^{-2}$ (Refs. 1 and 3) and whose impact grows with reduced thickness. The concentration gradient measured by both techniques shows a similar trend with lower values at the SOI/BOX interface, but the drop in the doping concentration predicted by the resistivity measurement is many orders of magnitude greater than that measured by SIMS. For device layer thicknesses lower than $20 \mathrm{~nm}$, the resistivity increased sharply. The grey region marked in the graph [see Fig. 3(a)] indicates the range of device layer thicknesses for

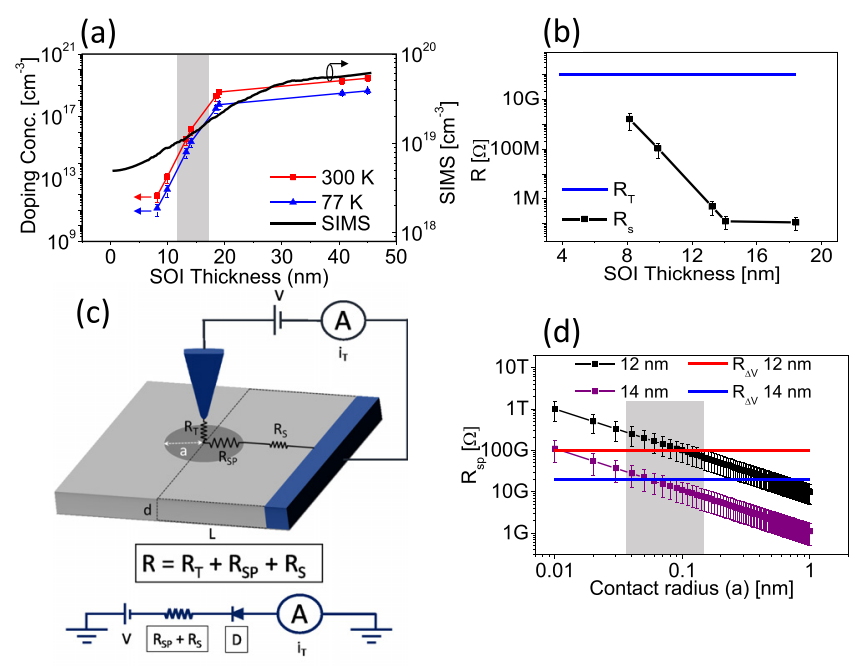

FIG. 3. (a) Doping concentration for SOIs for varying thicknesses calculated from the resistivity data measured at $300 \mathrm{~K}$ (red) and $77 \mathrm{~K}$ (blue). These values indicate that the electrically active dopant concentration reduces towards the $\mathrm{SOI} / \mathrm{BOX}$ interface, therefore deviating from the SIMS profile of phosphorous conc. (black) due to the interface trap states. (b) Tunneling resistance $R_{T}$ (blue), calculated at set points of $2 \mathrm{~V}$ and $20 \mathrm{pA}$, compared with sample resistance Rs (black), as a function of SOI thickness. (c) Schematic representation of tunneling in STM demonstrating the tip position over the sample, the effective tunneling area, and the resistive components such as tunneling resistance (Rt), spreading resistance (Rsp), and sample resistance (Rs). The effective tunneling area is indicated by the darker shade on the sample. A schematic representation of the STM circuit model ${ }^{1}$ is also presented. (d) The graph compares $\mathrm{R}_{\mathrm{SP}}$ calculated from the spreading resistance model with $\mathrm{R}_{\Delta \mathrm{V}}$ and the resistance calculated from the voltage blue shift observed in STM and STS. $R_{\text {SP }}$ calculated from the spreading resistance for $14 \mathrm{~nm}$ (purple) and $12 \mathrm{~nm}$ (black) SOIs is plotted with respect to the contact aperture radius. $R_{\Delta V}$ is shown in blue and red for 14 and $12 \mathrm{~nm}$, respectively. 
which a voltage shift was observed in STM and STS. A sharply increasing device layer resistivity for these thicknesses suggests a possible connection between the device layer resistivity and the STM/STS voltage shift. Figure 3(b) compares the tunneling resistance $\mathrm{R}_{\mathrm{T}}$ with the sample resistance $R_{S}$. Even though a sharp increase in $R_{S}$ is observed below $14 \mathrm{~nm}, R_{S}$ is still many orders of magnitude lower than $R_{T}$, and therefore, $R_{S}$ alone cannot be responsible for peak shifts in STM and STS.

Figure 3(c) shows a schematic of various resistive components of an STM. Figure 3(d) shows the model for the tunneling current circuit suggested by Johnson and Halbout, ${ }^{4}$ including the spreading resistance in the STM circuit. The spreading resistance $\left(\mathrm{R}_{\mathrm{SP}}\right)$ arises due to the current spreading radially out into the "bulk" of the sample from the point of 'contact' on the surface. $\mathrm{R}_{\mathrm{SP}}$ depends on the size of the contact and the resistivity $\rho$ of the sample. The relation is given by $\mathrm{R}_{\mathrm{SP}}=\frac{\rho}{4 a}$, where $a$ is the radius of the circular 'contact' area. ${ }^{5}$ In a typical STM sample holder configuration, $R_{S}$ and $R_{S P}$ along with $R_{T}$ add up in series to give a total resistance $\mathrm{R}$ [see Fig. 3(c)]. In an STM operation on a conductive sample, the voltage drop in the circuit is predominantly due to the tunneling resistance between the STM tip and the surface of the sample. Therefore, the tunneling resistance $R_{T}$, which is typically between 1 and $200 \mathrm{G} \Omega$, supersedes all other resistances in the circuit. However, for a high resistivity sample, the spreading resistance becomes comparable with the tunneling resistance and therefore results in a significant voltage drop in the sample locally near the tip, potentially causing the spectral shifts found in Fig. 1(a). Figure 3(d) compares $R_{\mathrm{SP}}$ calculated using the two equations $\mathrm{R}_{\mathrm{SP}}=\frac{\rho}{4 a}$ and $R_{\Delta \mathrm{V}}=\frac{\Delta \mathrm{V}}{\mathrm{I}}$, where $\mathrm{R}_{\Delta \mathrm{V}}$ is the resistance calculated from the voltage shift $(\Delta \mathrm{V})$ observed in STM and STS measurements and $I$ is the tunneling set-point current. In Fig. 3(d), $\mathrm{R}_{\mathrm{SP}}$ is calculated for $14 \mathrm{~nm}$ (purple) and $12 \mathrm{~nm}$ (black) SOIs using different values of $a$ from $0.1 \AA$ to $10 \AA$. $\mathrm{R}_{\mathrm{T}}$ calculated for the $14 \mathrm{~nm}$ sample (blue) SOI is around $20 \mathrm{G} \Omega$ and increases to between $75 \mathrm{G} \Omega$ and $100 \mathrm{G} \Omega$ for a $12 \mathrm{~nm}$ sample (red). We note that $\mathrm{R}_{\mathrm{T}}$ overlaps with $\mathrm{R}_{\mathrm{SP}}$ for the contact radius between $0.4 \AA$ and $1.5 \AA$ [indicated by the grey region marked in Fig. 3(d)] so that the effects we observe in STS and emission spectra can indeed be accounted for by the spreading resistance.

In order to verify these results, STM and STS measurements were repeated on SOI device layers with a higher dopant concentration. A $12 \mathrm{~nm}$ SOI sample with a doping concentration of $>10^{20} \mathrm{~cm}^{-3}$ was characterized with STM and STS at $77 \mathrm{~K}$. No voltage drop or shift in STS peaks were observed for these samples. Further measurements were also carried out on $5 \mathrm{~nm}$ thick samples by thinning the $12 \mathrm{~nm}$ SOI device layer. The image and the bias stability conditions are identical to those found on bulk $\mathrm{Si}(100)$. The STS spectra for the $5 \mathrm{~nm} \mathrm{SOI}$ is essentially identical to those of the bulk Si (100). These results demonstrate that with sufficient doping, the effect of the spreading resistance can be minimised to investigate the electronic structure of $5 \mathrm{~nm}$ thick device layers.

$\operatorname{Si}(100)$ device layers with different n-type doping concentrations were studied by STM and STS. The doping-level variation was obtained by thinning $45 \mathrm{~nm}$ thick SOI samples.
The dopant profile was obtained using ex-situ SIMS. We have demonstrated the sensitivity of STM/STS to the spreading resistance in $\mathrm{Si}(100)$ ultra-thin SOIs. We demonstrated the correlation between the dopant concentration and the voltage shift measured by STM and STS so that STS can be used to study the dopant concentration in the range of $10^{13}-10^{16} \mathrm{~cm}^{-3}$ in $\mathrm{Si}(100)$. The non-destructive and noncontact nature of the STM and STS reduce the non-linear effects arising due to the tip-surface interaction which can be predominant in typical contact based spreading resistance measurements. Furthermore, the high spatial resolution of STM/ STS is suitable for studying nano-structures, allowing effects due to buried interfaces, doping modulations, and leakage currents to be detected and characterized.

See supplementary material for the parameters used for the STS and field emission spectra and STM images showing the quality of the $12 \mathrm{~nm}$ SOI surface and PT in $14 \mathrm{~nm}$ SOI.

This work was supported by Science Foundation Ireland under PI Award Grant No. 12/IA/1482 and emanated from research supported in part by a research grant from Science Foundation Ireland (SFI) under Grant No. SFI/12/RC/2278.

${ }^{1}$ P. Zhang, E. Tevaarwerk, B. N. Park, D. E. Savage, G. K. Celler, I. Knezevic, P. G. Evans, M. A. Eriksson, and M. G. Lagally, Nature 439(7077), 703 (2006).

${ }^{2}$ K. C. Lin, O. W. Holland, L. C. Feldman, and H. H. Weitering, Appl. Phys. Lett. 72(18), 2313 (1998).

${ }^{3}$ P. Sutter, W. Ernst, and E. Sutter, Appl. Phys. Lett. 85(15), 3148 (2004).

${ }^{4}$ M. B. Johnson and J. M. Halbout, J. Vac. Sci. Technol., B 10(1), 508 (1992).

${ }^{5}$ R. G. Mazur and D. H. Dickey, J. Electrochem. Soc. 113(3), 255 (1966).

${ }^{6}$ R. M. Feenstra and J. A. Stroscio, J. Vac. Sci. Technol., B 5(4), 923 (1987).

${ }^{7}$ R. Maboudian, K. Pond, V. Bressler-Hill, M. Wassermeier, P. M. Petroff, G. A. D. Briggs, and W. H. Weinberg, Surf. Sci. 275(1), L662 (1992).

${ }^{8}$ N. D. Jäger, P. Ebert, K. Urban, R. Krause-Rehberg, and E. R. Weber, Phys. Rev. B 65(19), 195318 (2002).

${ }^{9}$ M. McEllistrem, G. Haase, D. Chen, and R. J. Hamers, Phys. Rev. Lett. 70(16), 2471 (1993).

${ }^{10}$ F. Giannazzo, F. Roccaforte, and V. Raineri, Appl. Phys. Lett. 91(20), 202104 (2007); P. Eyben, M. Xu, N. Duhayon, T. Clarysse, S. Callewaert, and W. Vandervorst, J. Vac. Sci. Technol., B: Microelectron. Nanometer Struct. Process., Meas., Phenom. 20(1), 471 (2002); E. Latu-Romain, P. Gilet, N. Chevalier, D. Mariolle, F. Bertin, G. Feuillet, G. PerillatMerceroz, P. Ferret, F. Levy, P. Muret, and A. Chelnokov, J. Appl. Phys. 107(12), 124307 (2010).

${ }^{11}$ D. Álvarez, J. Hartwich, J. Kretz, M. Fouchier, and W. Vandervorst, Microelectron. Eng. 67-68, 945 (2003).

${ }^{12}$ F. Giannazzo, V. Raineri, S. Mirabella, G. Impellizzeri, and F. Priolo, Appl. Phys. Lett. 88(4), 043117 (2006); L. Wang, V. Sallet, C. Sartel, and G. Brémond, ibid. 109(9), 092101 (2016); G. Bremond, L. Wang, J.-M. Chauveau, C. Sartel, and V. Sallet, in Proceedings of the European Microscopy Congress 2016 (Wiley-VCH Verlag GmbH \& Co. KGaA, 2016); F. Giannazzo, V. Raineri, S. Mirabella, G. Impellizzeri, and F. Priolo, Microelectron. Eng. 84(3), 446 (2007).

${ }^{13}$ A. Schulze, R. Cao, P. Eyben, T. Hantschel, and W. Vandervorst, Ultramicroscopy 161, 59 (2016); K. Mylvaganam, L. C. Zhang, P. Eyben, J. Mody, and W. Vandervorst, Nanotechnology 20(30), 305705 (2009).

${ }^{14}$ P. S. N. Barimar, C. M. Doyle, B. Naydenov, and J. J. Boland, J. Vac. Sci. Technol., B 34(4), 041806 (2016).

${ }^{15}$ B. Naydenov and J. J. Boland, Phys. Rev. B 82(24), 245411 (2010).

${ }^{16}$ R. S. Becker, J. A. Golovchenko, and B. S. Swartzentruber, Phys. Rev. Lett. 55(9), 987 (1985); G. Binnig, K. H. Frank, H. Fuchs, N. Garcia, B. Reihl, H. Rohrer, F. Salvan, and A. R. Williams, ibid. 55(9), 991 (1985).

${ }^{17}$ J. A. Appelbaum, G. A. Baraff, and D. R. Hamann, Phys. Rev. B 14(2), 588 (1976); J. Pollmann, P. Krüger, and A. Mazur, J. Vac. Sci. Technol., B 
5(4), 945 (1987); J. A. Kubby and J. J. Boland, Surf. Sci. Rep. 26(3), 61 (1996).

${ }^{18}$ T. Jung, Y. W. Mo, and F. J. Himpsel, Phys. Rev. Lett. 74(9), 1641 (1995); C. L. Lin, S. M. Lu, W. B. Su, H. T. Shih, B. F. Wu, Y. D. Yao, C. S. Chang, and T. T. Tsong, ibid. 99(21), 216103 (2007); H.-C. Ploigt, C. Brun, M. Pivetta, F. Patthey, and W.-D. Schneider, Phys. Rev. B 76(19), 195404 (2007); P. Ruffieux, K. Ait-Mansour, A. Bendounan, R. Fasel, L. Patthey, P. Groning, and O. Groning, Phys. Rev. Lett. 102(8), 086807 (2009).
${ }^{19}$ W. J. Kaiser, L. D. Bell, M. H. Hecht, and F. J. Grunthaner, J. Vac. Sci. Technol., A 6(2), 519 (1988); M. Weimer, J. Kramar, and J. D. Baldeschwieler, Phys. Rev. B 39(8), 5572 (1989).

${ }^{20}$ N. D. Jäger, E. R. Weber, K. Urban, and P. Ebert, Phys. Rev. B 67(16), 165327 (2003).

${ }^{21}$ F. J. Himpsel and T. Fauster, J. Vac. Sci. Technol., A 2(2), 815 (1984).

${ }^{22}$ S. M. Sze and K. K. Ng, Physics of Semiconductor Devices (John Wiley \& Sons, 2006).

${ }^{23}$ C. Ndoye, T. Liu, and M. Orlowski, ECS Trans. 33(31), 3 (2011). 